\title{
Temporal Relation between Disappearance of Penumbral Fine-Scale Structure and Evershed Flow
}

\author{
M. Kubo \\ National Astronomical Observatory, Mitaka, Tokyo, 181-8588, Japan. \\ masahito.kubo@nao.ac.jp \\ K. Ichimoto \\ Hida Observatory, Kyoto University, Takayama, Gifu 506-1314, Japan. \\ B. W. Lites \\ High Altitude Observatory, National Center for Atmospheric Research, P.O. Box 3000, \\ Boulder, CO 8030\%1. \\ R. A. Shine \\ Lockheed Martin Solar and Astrophysics Laboratory, Building 252, 3251 Hanover Street, \\ Palo Alto, CA 94304.
}

\begin{abstract}
We investigate the temporal relation between the Evershed flow, dot-like bright features (penumbral grain), the complex magnetic field structure, and dark lanes (dark core) along bright filaments in a sunspot penumbra. We use a time series of high spatial resolution photospheric intensity, vector magnetic field maps, and Doppler velocity maps obtained with the Solar Optical Telescope aboard the Hinode spacecraft. We conclude that the appearance and disappearance of the Evershed flow and penumbra grains occur at nearly the same time and are associated with changes of the inclination angle of the magnetic field from vertical to more horizontal. This supports the idea that Evershed flow is a result of thermal convection in the inclined field lines. The dark core of the bright penumbral filament also appears coincidental with the Evershed flow. However, the dark-cored bright filament survives at least for 10-20 minutes after the disappearance of the Evershed flow. The heat input into the bright filament continues
\end{abstract}

\footnotetext{
${ }^{1}$ The National Center for Atmospheric Research is sponsored by the National Science Foundation
} 
after the end of heat transfer by the Evershed flow. This suggests that local heating along the bright filament is important to maintain the brightness of the bright filament in addition to the heat transfer by the Evershed flow.

Subject headings: Sun: magnetic fields — Sun: photosphere — (Sun:) sunspots

\section{INTRODUCTION}

Sunspot penumbrae consist of many fine-scale radial filaments. Their fine-scale structures reflect complex magnetic field structures in penumbrae: azimuthal fluctuations of the penumbral magnetic field inclination and field strength are well observed at the scale of the intensity fluctuations (e.g. Degenhardt \& Wiehr 1991; Schmidt et al. 1992; Title et al. 1993; Lites et al. 1993). A systematic outward flow called Evershed flow is important for understanding the formation of such complex magnetic filed structures in penumbrae. Evershed flows are observed as radial filamentary structures of Doppler blueshifts at the disk-center side penumbra, and Doppler redshifts at the limb side penumbra. The relation between penumbral fine-scale structures and Evershed flows have been investigated by numerous authors for a long period, and recent observations with a high spatial resolution clearly confirms the following properties (e.g. Bellot Rubio et al. 2004; Langhans et al. 2005; Rimmele \& Marino 2006; Ichimoto et al. 2007a): (1) The Evershed flow is a nearly horizontal outward flow observed with horizontal magnetic field component in the penumbra. (2) The horizontal field component is observed along the bright filament in the inner penumbra, and the dark filament in the outer penumbra. (3) The Evershed flow originates at a dot-like bright feature called a penumbral grain in the inner penumbra. These observational results suggest that Evershed flows are radial outward flows propagating along the penumbral horizontal magnetic fields, and their origin is rising hot gas well observed in the inner penumbra. The formations of Evershed flows and penumbral fine-scale magnetic field structures are closely related each other.

Many models have been proposed to explain penumbral fine-scale structures and Evershed flows, two major ones are an "uncombed" penumbral model (Solanki \& Montavon 1993) and a "gappy" penumbral model (Spruit \& Scharmer 2006; Scharmer \& Spruit 2006). A nearly horizontal flux tube is embedded in more vertical background fields in the uncombed penumbral model. On the basis of the this model, Schlichenmaier et al. (1998) proposed a moving flux tube model, in which a thin flux tube carrying hot Evershed flow rises into the background fields and moves toward the umbra. In the gappy penumbral model, a cusp-like magnetic field structure is formed by convection in field-free gaps just below the visible solar surface. Recent 3D magnetohydrodynamic (MHD) simulations with radiative transfer are 
beginning to produce penumbral filaments with a systematic outflow like observed sunspot penumbrae (Heinemann et al. 2007; Rempel et al. 2009). From such 3D MHD simulations, a kind of hybrid model is proposed that explains the Evershed effect as a natural consequence of "convection" in strong, inclined magnetic fields (Scharmer et al. 2008; Rempel et al. 2009; Kitiashvili et al. 2009). Evershed flows are associated with weaker and horizontal magnetic fields in the deep photospheric layer (e.g. Jurcák et al. 2007; Borrero et al. 2008), but the field-free plasma has not yet been detected near the $\tau=1$ surface in the penumbra even with spectropolarimetric measurements at the current highest spatial resolution (Borrero \& Solanki 2008). Moreover, Ichimoto et al. (2007b) have discovered thin inclined dark stripes along the bright penumbral filaments suggesting overturning perpendicular convection within the bright penumbral filaments.

In this study, we investigate the temporal relation between the Evershed flow, a penumbral grain, the complex magnetic field structure, and a bright penumbral filament. However, it is not easy to trace a penumbral filament from its birth to death because penumbral filaments and Evershed flows are always changing everywhere in the penumbra. We use the dark core of a bright filament in the penumbra in order to trace the center of the bright filament. Such dark cores were discovered as narrow dark lanes with a width less than $200 \mathrm{~km}$ in the center of bright penumbral filaments (Scharmer et al. 2002). The dark core is thought to be a result of a cusp shape of the $\tau=1$ surface (Spruit \& Scharmer 2006; Schüssler \& Vögler 2006; Heinemann et al. 2007; Rempel et al. 2009). A pile-up of hot plasma along the center of the bright penumbral filament pushes up the the $\tau=1$ surface into the upper layer, where the temperature is lower. As a result, a dark lane is observed along the top of the cusp of the $\tau=1$ surface. Rimmele (2008) has reported that the dark cores are more clearly observed in the line core than in the continuum, and suggested that the dark cores form in the upper layer of the photosphere.

The dark-cored bright filaments in the penumbra are not transient phenomena, and they often have lifetimes on the order of 1-2 hr (Langhans et al. 2007). The space-borne observations with the Solar Optical Telescope (SOT; Tsuneta et al. 2008) aboard the Hinode spacecraft (Kosugi et al. 2007) have an advantage in observing the detailed evolution of the penumbral fine-scale structures and Evershed flows during their lifetimes. We can identify a dark core with shorter wavelengths of the SOT, but the $0.3^{\prime \prime}$ spatial resolution of the SOT spectropolarimeter is not enough to investigate the magnetic field vector of dark cores. The nature of dark cores is not the target of this paper. Our goal is to investigate the nature of the magnetoconvection in the penumbra by tracing the dark core. 


\section{OBSERVATION AND DATA ANALYSIS}

The SOT observations of a sunspot in NOAA AR 10944 on 2007 February 27 and calibration procedures for the observed data were described in Kubo et al. (2008). In this Letter, we compare maps of the physical parameters obtained by the spectropolarimeter (SP) to G-band images with the broadband filter imager (BFI), as shown in Figure 1. The G-band images were obtained with a cadence of 2 minutes and a pixel sampling of $0.054^{\prime \prime}$. The SP repeatedly obtained spatial distributions of Stokes profiles with a field of view of $9.5^{\prime \prime} \times 82^{\prime \prime}$ every 5.5 minutes. The spatial sampling of SP was $0.149^{\prime \prime} \times 0.160^{\prime \prime}$. The cadence of 5.5 minutes for the SP is insufficient to trace an individual Evershed flow (cloud). However, a penumbral filament is more likely to drift out of the field-of-view during its lifetime for a higher cadence because of the corresponding narrower field-of-view required.

The magnetic field vector and Doppler velocity in the photosphere were derived from Stokes profiles of two Fe I lines at 630.15 and $630.25 \mathrm{~nm}$ by assuming a Milne-Eddington representation of the atmospheric stratification and a two-component model atmosphere (a magnetized atmosphere and a non-magnetized atmosphere). We investigated the magnetic field strength, the magnetic field inclination with respect to the line-of-sight direction, the Doppler velocity, the continuum intensity, and the intensity at the center of the Fe I $\lambda$ 6301.5 line. An inclination angle of $90^{\circ}$ is defined as a magnetic field perpendicular to the line-of-sight direction. Because the sunspot is located close to the disk center (S01, E13), the line-of-sight direction is almost parallel with the normal direction to the solar surface. This is good for investigating the interlaced vertical and horizontal components of penumbral magnetic fields. The reference point of the Doppler velocity in each map is the average Doppler velocity in the umbra. The continuum and line center intensities are normalized to the average continuum intensities in the quiet area outside the sunspot.

The G-band images are also normalized to average intensities in the quiet area. We aligned the sunspot centers in sequential G-band images in order to remove any drifts due to the correlation tracker (Shimizu et al. 2008) and any sunspot proper motion. The sunspot center is determined as the center-of-gravity of the G-band intensity in the umbra. The maps obtained by the SP were aligned to the G-band images taken at the time closest to that of the midpoint of the SP maps, using the image cross-correlation between the line center intensity maps and the G-band images.

\section{RESULTS}

Our target dark core (in a bright penumbral filament) is indicated by the white arrow in Figure 2 $a$. The dark core originates at a penumbral grain (the black arrow in Fig. 2 $2 a$ ). The dark-

cored penumbral filament that is resolved in the G-band image can be identified in the line center intensity map of the SP, but it is hard to identify in the continuum intensity map (Figs. $22 b$ and $2 c$ ). The difference between dark-cored penumbral filaments as observed in the line core and continuum 
intensity has been already reported by Rimmele (2008).

Figure $2 d$ shows that the dark core has a larger blueshift than its lateral surroundings. Since it is located in the center-side penumbra, this larger blueshift corresponds to the Evershed flow (cloud). The sunspot is located near the disk center at $(\mu>0.96)$. Therefore the nearly horizontal Evershed flow has a small line of sight component, but we can still clearly identify its contribution. The magnetic fields generally become more horizontal with increasing distance from the sunspot center over the whole penumbra (Fig 2 2 ). The more horizontal field in the dark core is also weaker than either side as seen in Figures $2 e$ and $2 f f$. This dark core is larger than a typical width of $90 \mathrm{~km}$ (Scharmer et al. 2002). On the other hand, the properties of magnetic field vector and Doppler velocity observed in our dark core are very similar to those in the previous studies (Bellot Rubio et al. 2005; Langhans et al. 2007; Bellot Rubio et al. 2007). We therefore assume that our target, although larger than average, is an otherwise ordinary dark-cored penumbral filament.

We hereafter focus on the evolution of the dark-cored penumbral filament. The dark core is shown by the dotted lines in Figure $3 a$ which were manually determined for each image. The dark core elongates into the umbra in concert with the inward motion of the penumbral grain from 11:53UT to 12:38UT. The dark core breaks into two parts, and the inner (umbra-side) part shrinks into the umbra from 12:49UT to 13:11UT. The outer part again elongates into the umbra with another inward migrating penumbral grain from 13:22UT to 13:44UT, and then the dark core gradually becomes narrower and less dark from 13:44UT. Finally, no dark core is visible in the frame at $14: 18 \mathrm{UT}$.

Figure $4 a$ shows the temporal evolution of the G-band intensity along the dotted lines in Figure $3 a$. This shows two inward moving penumbral grains, and dark areas (dark cores) which also move into the umbra. The Evershed flows also originate at the penumbral grains (Fig. $4 b$ ). It is not observed between the two successive penumbral grains around 12:45UT and it stops at 13:45 with the disappearance of the second penumbral grain. Small redshifts are observed along the dark-cored bright filament in the frame at 13:56 in Figure 3b. One interesting result is that the dark core is still observed until 14:12 after the disappearance of the Evershed flow. Figure 4 $c$ shows that the areas with more horizontal fields move a short distance toward the umbra during the period when Evershed flows are observed (the white arrows) in comparison to the period without any Evershed flows (the black arrows). The magnetic fields at the dark core return to the same orientation as their surroundings (more vertical) just after the penumbral grain and Evershed flow are no longer observed along the dark core. Figure 3 $c$ shows that the more horizontal fields are observed along the dark core at 13:44 but not seen at 13:56. The 2D distribution of magnetic field inclination is not much changed after 13:00, and the area with vertical fields suddenly appears along the dark core in the period between 13:44 and 13:56. Higher time resolution is necessary to reveal the process of the disappearance of fine-scale magnetic field structures in the penumbra.

Another dark-cored penumbral filament (the yellow arrows in Fig 3) and a dark penumbral fil- 
ament (the green arrows) are observed in the same field-of-view. These dark features also have more horizontal fields than their lateral surroundings, and have similar spatial and temporal relations with the Evershed flows.

\section{DISCUSSIONS}

We confirm that the Evershed flow originates at penumbral grains and is associated with a more horizontal magnetic field. In this paper we describe a bright penumbral filament with a dark core that survives for 10-20 minutes beyond the end of the Evershed flows. This means that the convective upflow along the center of the bright penumbral filament, which produces warping of the $\tau=1$ surface (i.e., the dark core), continues after the disappearance of the Evershed flow, since a cooling time scale in the photosphere is much shorter than 10-20 minutes (Schlichenmaier et al. 1999). The disappearance of the Evershed flow is locally accompanied by a change of magnetic field inclination to more vertical. The absence of the Evershed flow associated with convection in vertical fields suggests that both convection and horizontal fields in the penumbra are necessary for (or caused by) the formation of the Evershed flow. The close correlation between the Evershed flow, convection, and more horizontal fields supports recent models wherein the Evershed flows are convective flows in the inclined magnetic field lines in the penumbra (Scharmer et al. 2008; Rempel et al. 2009; Kitiashvili et al. 2009).

If the Evershed flow only caused a pile-up of hot plasma along the bright filament, the dark core should disappear within a cooling time scale after the end of the Evershed flow. However, the dark core is continuously observed without the Evershed flow for a period longer than a cooling time scale in the photosphere. This suggests that a heat source, in addition to the heat transfer by the Evershed flows, is necessary to form a bright filament with a dark core. It is believed that the Evershed flows are radially outward flows along the horizontal fields from the inner penumbra (penumbral grains) to the outer penumbra. They may carry the energy through the penumbra in the horizontal direction rather than the vertical direction. Although it may be hard to identify the Evershed flows from convection in the penumbra, one possible source of the heat for the bright penumbral filament is vertical convective motions locally formed along the bright filament rather than the larger scale heat transfer due to the Evershed flows.

A major remaining issue is the vertical stratifications in the penumbral fine-scale structures. After the Evershed flow disappears in the observed layer, it may remain in the deeper photosphere or below the photosphere. Recently, signatures of convection (i.e. downward and upward motions)

in umbral dots (Ortiz et al. 2010) and light bridges (Rouppe van der Voort et al. 2010) are reported only in the deep photosphere from a bisector analysis of the Fe I $630 \mathrm{~nm}$ lines taken with the CRISP at the Swedish $1 \mathrm{~m}$ Solar Telescope. However, the signature of convective downflows is not detected from the same analysis of Fe I $709 \mathrm{~nm}$ line taken with the Littrow spectrograph at the same telescope (Bellot Rubio et al. 2010). It is still unknown how the Evershed flows in the deeper layer affect 
the formation of the convection in the observed layer and heating of bright filaments. Moreover, the evolution of the vertical stratifications is essential for understanding the formation and decay of the penumbral complex magnetic field structures. We have observed redshifts along the dark core when the more horizontal magnetic field disappears from the dark-cored penumbral filament, but they are very small. It is not clear if the observed redshifts correspond to the downward motion of the magnetic field lines because a flow along the field lines also can produce such a small redshift. If the more horizontal fields have either upward motion or downward motion during their disappearance, the vertical stratifications of the magnetic field vector should give us information about their motion.

We would like to thank J. M. Borrero, M. Rempel, R. Schlichenmaier, Y. Katsukawa, A. Lagg, J. Jurčák, T. Magara, and S. Tsuneta for fruitful comments. Hinode is a Japanese mission developed and launched by ISAS/JAXA, with NAOJ as domestic partner and NASA and STFC (UK) as international partners. It is operated by these agencies in co-operation with ESA and NSC Norway. This work was partly carried out at the NAOJ Hinode Science Center, which is supported by the Grant-in-Aid for Creative Scientific Research "The Basic Study of Space Weather Prediction" from MEXT, Japan (Head Investigator: K. Shibata), generous donations from Sun Microsystems, and NAOJ internal funding.

\section{REFERENCES}

Bellot Rubio, L. R., Balthasar, H., \& Collados, M. 2004, A\&A, 427, 319

Bellot Rubio, L. R., Langhans, K., \& Schlichenmaier, R. 2005, A\&A, 443, L7

Bellot Rubio, L. R., et al. 2007, ApJ, 668, L91

Bellot Rubio, L. R., Schlichenmaier, R., \& Langhans, K. 2010, ApJ, 725, 11

Borrero, J. M. 2007, A\&A, 471, 967

Borrero, J. M., Lites, B. W., \& Solanki, S. K. 2008, A\&A, 481, L13

Borrero, J. M., \& Solanki, S. K. 2008, ApJ, 687, 668

Degenhardt, D., \& Wiehr, E. 1991, A\&A, 252, 821

Heinemann, T., Nordlund, Å., Scharmer, G. B., \& Spruit, H. C. 2007, ApJ, 669, 1390

Ichimoto, K., et al. 2007, PASJ, 59, 593

Ichimoto, K., et al. 2007, Science, 318, 1597

Jurcák, J., et al. 2007, PASJ, 59, 601 
Kitiashvili, I. N., Kosovichev, A. G., Wray, A. A., \& Mansour, N. N. 2009, ApJ, 700, L178

Kosugi, T., et al. 2007, Sol. Phys., 243, 3

Kubo, M., et al. 2008, ApJ, 681, 1677

Langhans, K., Scharmer, G. B., Kiselman, D., Löfdahl, M. G., \& Berger, T. E. 2005, A\&A, 436, 1087

Langhans, K., Scharmer, G. B., Kiselman, D., \& Löfdahl, M. G. 2007, A\&A, 464, 763

Lites, B. W., Elmore, D. F., Seagraves, P., \& Skumanich, A. P. 1993, ApJ, 418, 928

Ortiz, A., Bellot Rubio, L. R., \& Rouppe van der Voort, L. 2010, ApJ, 713, 1282

Rempel, M., Schüssler, M., \& Knölker, M. 2009, ApJ, 691, 640

Rimmele, T., \& Marino, J. 2006, ApJ, 646, 593

Rimmele, T. 2008, ApJ, 672, 684

Rouppe van der Voort, L., Bellot Rubio, L. R., \& Ortiz, A. 2010, ApJ, 718, L78

Scharmer, G. B., Gudiksen, B. V., Kiselman, D., Löfdahl, M. G., \& Rouppe van der Voort, L. H. M. 2002, Nature, 420, 151

Scharmer, G. B., \& Spruit, H. C. 2006, A\&A, 460, 605

Scharmer, G. B., Nordlund, Å., \& Heinemann, T. 2008, ApJ, 677, L149

Schmidt, W., Hofmann, A., Balthasar, H., Tarbell, T. D., \& Frank, Z. A. 1992, A\&A, 264, L27

Schlichenmaier, R., Jahn, K., \& Schmidt, H. U. 1998, A\&A, 337, 897

Schlichenmaier, R., Bruls, J. H. M. J., \& Schüssler, M. 1999, A\&A, 349, 961

Schüssler, M., \& Vögler, A. 2006, ApJ, 641, L73

Shimizu, T., et al. 2008, Sol. Phys., 249, 221

Sobotka, M., Brandt, P. ., \& Simon, G. W. 1999, A\&A, 348, 621

Solanki, S. K., \& Montavon, C. A. P. 1993, A\&A, 275, 283

Spruit, H. C., \& Scharmer, G. B. 2006, A\&A, 447, 343

Sütterlin, P., Bellot Rubio, L. R., \& Schlichenmaier, R. 2004, A\&A, 424, 1049

Title, A. M., Frank, Z. A., Shine, R. A., Tarbell, T. D., Topka, K. P., Scharmer, G., \& Schmidt, W. 1993, ApJ, 403, 780 
Tsuneta, S., et al. 2008, Sol. Phys., 249, 167

This preprint was prepared with the AAS LATEX macros v5.2. 


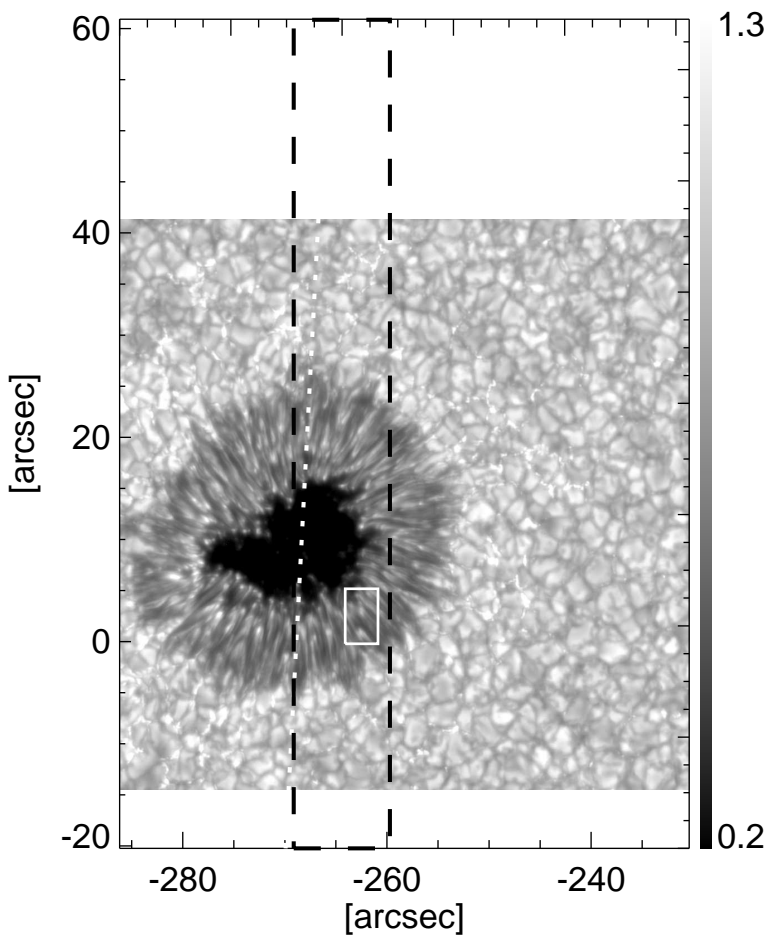

Fig. 1.- G-band image of a sunspot in NOAA AR 10944 on 2007 February 27. The dashed box shows the field-of-view of the Hinode SP and is identical to the field-of-view of Fig. 2 , The dotted line is the boundary dividing the limb-side and center-side penumbra. The grayscale bar shows the intensity level normalized to the mean intensity of the quiet area outside the sunspot. The horizontal and vertical axes represent the positions with respect to the disk center. 

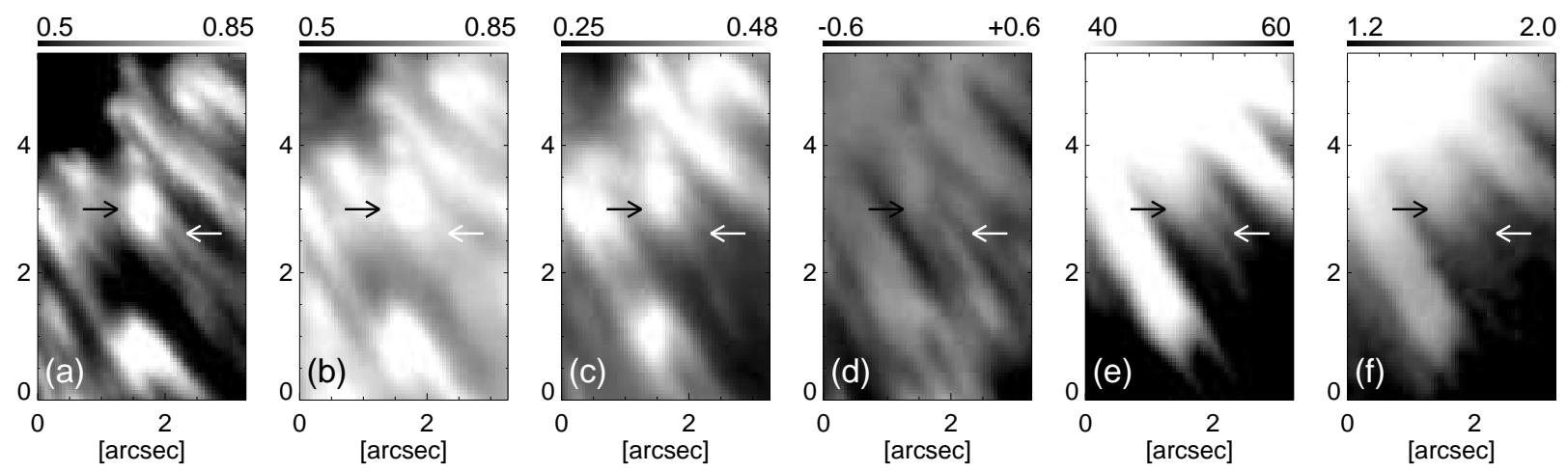

Fig. 2.- A penumbral grain (the black arrow) and a dark core of the bright penumbral filament (the white arrow) in the box of Fig. 1. (a) G-band image, (b) continuum intensity map, $(c)$ line center intensity map, $(d)$ Doppler velocity map, $(e)$ inclination map, and $(f)$ field strength map at 11:53UT on 2007 February 27. The positive and negative Doppler velocity indicates a redshift and blueshift in units of $\mathrm{km} \mathrm{s}^{-1}$, respectively. The magnetic field with the line-of-sight direction is represented by the inclination angle of $0^{\circ}$. The unit of the magnetic field strength is $10^{3}$ Gauss. 

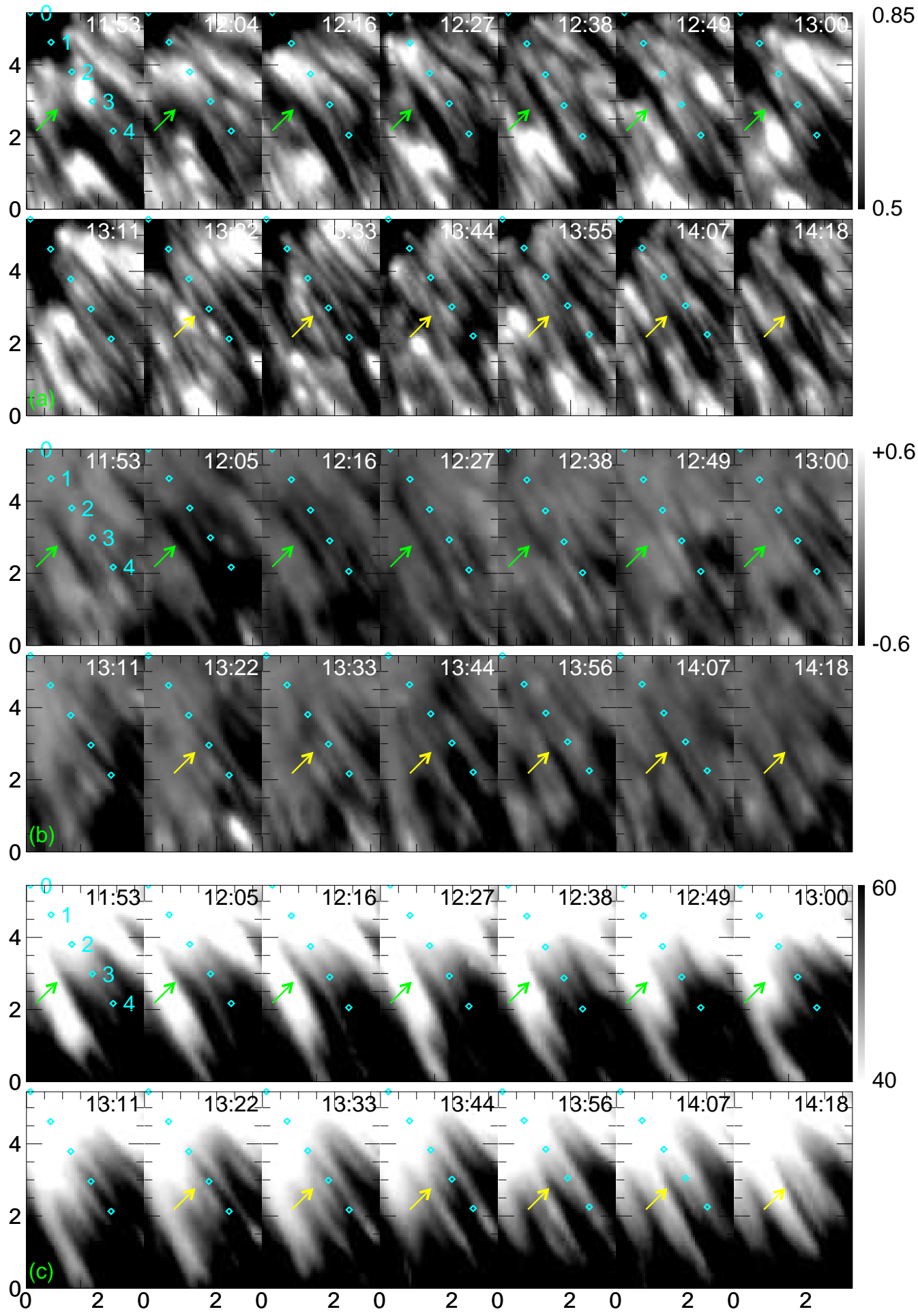

Fig. 3.- Time series of (a) G-band images, (b) Doppler velocity maps, and (c) inclination maps in the box of Fig. 1. The upper left panel in each set is identical to the corresponding signal in Figure 2. The times in panels $(b)$ and $(c)$ are for the midpoint of each SP map. The dots with intervals of $1^{\prime \prime}$ are linearly aligned on a dark core in the bright penumbral filament. The scales are in units of arcseconds. 

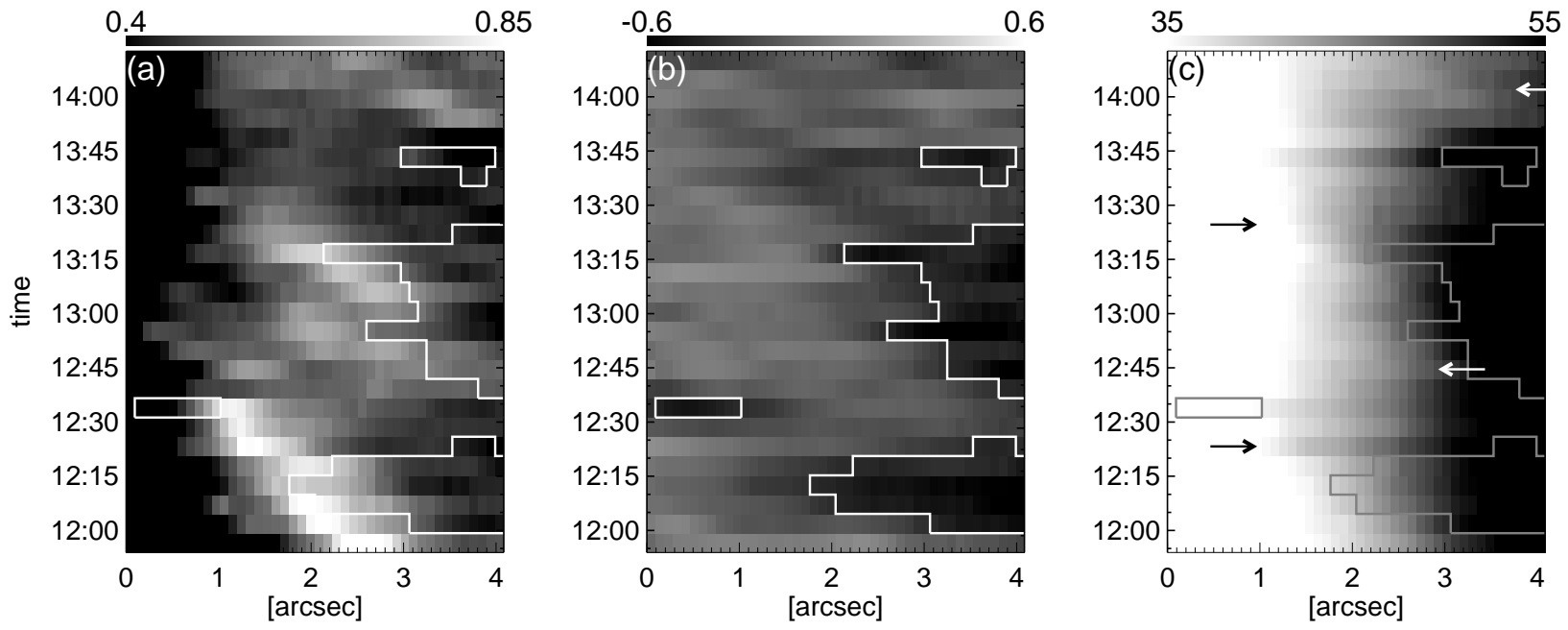

Fig. 4.- Space vs. time plots along the dotted lines in Fig. 3 for (a) G-band intensity, (b) Doppler velocity, and (c) magnetic field inclination from 11:53UT to 14:12UT on 2007 February 27. The cadence is 5.5 minutes. The dot at the top of each map in Fig. 3 corresponds to $0^{\prime \prime}$ of the horizontal axis. The contours represent the Doppler velocity of $-0.4 \mathrm{~km}$ $\mathrm{s}^{-1}$. Note that the dark core is observed until 14:12UT, and not observed at 14:18UT. 